\title{
Kenebo shipibo-conibo y noción de espacio topológico en niños de 5 años
}

\author{
Wendy Nelly Bada Laura ${ }^{1}$ \\ Ministerio de Educación Nacional del Perú, Perú
}

Autor de correspondencia: ${ }^{1}$ wendybadalaura@yahoo.com Recibido: 17 de diciembre de 2020 Revisado: 13 enero de 2021 Aprobado: 12 de abril de 2021 Publicado: 19 de septiembre de 2021

\section{Resumen}

Para los shipibo-conibo, la visión de los diseños - o keneboes fundamental para entender su relación con lo natural, con lo espiritual y con la noción de su ser en el espacio y tiempo. Para algunos autores, la noción del espacio (NE) en niños de educación inicial mejora con el tiempo y depende del entorno. El objetivo del estudio es determinar la influencia de los kenebos shipibo-conibo sobre la NE en niños de educación inicial. Metodológicamente, la investigación es cuantitativa, aplicada, descriptiva y experimental. La población consistió en 48 niños de dos instituciones educativas de Yarinacocha, Ucayali, Perú, en 2019. Se formaron dos grupos de 24 niños - control y experimental-. El segundo fue intervenido con talleres sobre kenebos shipibo-conibo y con una guía de observación. Se midió el puntaje de competencias sobre la NE en cada grupo con un diseño experimental y pre- y postest. El tratamiento estadístico fue descriptivo e inferencial, basado en medidas de tendencia central y de dispersión. Para la comparación de medianas, se utilizó la prueba U de Mann-Whitney. Para analizar las diferencias entre las distribuciones de las calificaciones, se utilizó la prueba de Kolmogorov-Smirnov. En los resultados se determinó que la intervención fue efectiva. Se concluye que el kenebo shipiboconibo influyó significativamente en las capacidades relativas a la NE.

Palabras clave: educación intercultural, taller educativo, espacio, pedagogía, kenebo, shipibo-conibo 


\title{
Shipibo-Conibo kenebo and the notion of topological space in 5-year-old children
}

\begin{abstract}
For the Shipibo-Conibo, the view of the designs-or "kenebo"-is fundamental to understanding their relationship with the natural, the spiritual, and the notion of their being in space and time. For some authors, the notion of space (NS) in pre-school children improves over time and depends on the environment. The aim of the study is to determine the influence of the Shipibo-Conibo designs on the NS in pre-school children. Methodologically, the research is quantitative, applied, descriptive, and experimental. The sample consisted of 48 children from two educational institutions in Yarinacocha, Ucayali, Peru, in 2019. Two groups of 24 children-control and experimental-were formed. The latter was intervened with workshops on Shipibo-Conibo kenebos and with an observation guide. The competence score on the NS was measured in each group, with an experimental design and pre- and posttests. The statistical analysis was descriptive and inferential, based on measures of both central tendency and of dispersion. For the comparison of medians, the Mann-Whitney U test was used. For the analysis of differences between the distributions of scores, the KolmogorovSmirnov test was used. The results found that the intervention was effective. It is concluded that the Shipibo-Conibo kenebo had a significant influence on the abilities related to the NS.
\end{abstract}

Keywords: intercultural education, educational workshop, space, pedagogy, kenebo, Shipibo-Conibo

\section{Kenebo shipibo-conibo e noção de espaço topológico em crianças de 5 anos}

\section{Resumo}

Para os shipibo-conibo, a visão dos desenhos - ou "kenebo" - é fundamental para entender sua relação com o natural, o espiritual e a noção de seu ser no espaço e no tempo. Para alguns autores, a noção de espaço (NE) em crianças pré-escolares melhora com o tempo e depende do meio ambiente. $\mathrm{O}$ objetivo do estudo é determinar a influência do kenebo shipibo-conibo sobre a NE em crianças em idade pré-escolar. Metodologicamente, a pesquisa é quantitativa, aplicada, descritiva e experimental. A amostra consistiu em 48 crianças de duas instituições educacionais em Yarinacocha, Ucayali, Peru, em 2019. Dois grupos de 24 crianças - controle e experimental - foram formados. O segundo grupo foi intervindo com oficinas sobre kenebos shipibo-conibo e com um guia de observação. A pontuação das competências sobre a NE foi medida em cada grupo, com um desenho experimental com um pré e um pós-teste. A análise estatística foi descritiva e inferencial, baseada em medidas tanto de tendência central como de dispersão. Para a comparação das medianas, foi utilizado o teste Mann-Whitney U. Para a análise das diferenças entre as distribuições de pontuação, foi utilizado o teste Kolmogorov-Smirnov. Os resultados constataram que a intervenção foi eficaz. Conclui-se que o kenebo shipibo-conibo teve uma influência significativa nas habilidades relacionadas com a NE.

Palavras-chave: educação intercultural, oficina educativa, espaço, pedagogia, kenebo, Shipibo-Conibo 
En el desarrollo de una educación inicial hay diferentes actores imprescindibles para conseguir capacidades, destrezas y habilidades en los niños, y en consecuencia una educación de calidad. Los espacios adecuados brindan interacción entre niños, docentes y padres de familia, lo cual propicia experiencias y aprendizajes significativos (Morales, 2014). En este contexto, toda persona nace en una zona delimitada por diversidad de cosas y en conjunto con otros individuos con los que interactúa por medio de los sentidos. En referencia a los niños en fase de educación inicial, ellos están condicionados por "las prácticas que diariamente vive[n]" (Castro, 2004. p. 163).

Hoy la educación inicial es el primer nivel de la educación básica regular y atiende a niños menores de 6 años. Se instituye en dos ciclos: el primero para niños y niñas menores de 3 años y el segundo para niños y niñas de 3 a 5 años. Este periodo es primordial para la vida del niño, debido a que en los primeros seis años construyen las raíces de su progreso integral y ulteriores aprendizajes. Por tal motivo, se necesitan educadores que acepten el desafío de producir planteamientos educativos innovadores, que integren las sapiencias y las exploraciones en pro de los niños y niñas menores de seis años; que admitan y consideren sus particularidades, sus requerimientos e inclinaciones, con el propósito de avivar el pleno avance de sus aptitudes y de aprendizajes significativos a partir de prácticas lúdicas, creativas y de intercambio, en una esfera de amor y seguridad (Vargas, 2018).

En el progreso cognitivo de los niños y niñas, el concepto de espacio es fundamental para lograr el desarrollo que le ayudará al entendimiento de su entorno, a su orientación, a la descripción de lugares y a leer correctamente ubicaciones y mapas. En la noción espacial de los individuos, el despliegue cognitivo comprende una sostenida y paulatina consecución de habilidades que admitan su acrecentamiento. Esta noción avanza desde la ausencia de percepción de la existencia de relaciones espaciales entre objetos; continúa con el manejo topológico, donde se capta el espacio perceptual y egocéntrico; y finaliza con la utilización del sistema de coordenadas y la perspectiva de operaciones concretas (Rimassa \& Fernández-Silva, 2014).

El aprendizaje de los niños en sus primeros años de vivencia escolar se caracteriza por la actividad física, con una permanente interacción con su medio y la investigación constante, derivada de su propia intuición, que los lleva a buscar explicaciones con la construcción y desarrollo del pensamiento simbólico y concreto. Por ello, en estos primeros años se desarrollan las habilidades y competencias que los llevan a comprender el concepto de espacio (Castro, 2004). Por su lado, Fernández y Ramiro (2015) exponen que concebir y manejar la concepción de espacio representa el haber alcanzado una sucesión de nociones espaciales (NE) que muestran los diversos lugares y colocaciones que posee un objeto o un cuerpo. En tal sentido, ayudar y operar la significación de espacio empiezan por apoyar al niño a tener consciencia de estas nociones. A la fase pedagógica de educación infantil le corresponde asistir con distintas operaciones para formar y lograr cimientos fuertes.

Según los autores citados, en la educación inicial se debe instruir al niño en las siguientes NE:

- La orientación de un objeto o del propio cuerpo, ya que todo objeto se orienta a través de tres dimensiones: la lateralidad —izquierda o derecha-, la profundidad delante o detrás- y la anterioridad —antes y después-. 
- La posición en el espacio con relación a la interioridad - estar dentro de un espacioo la exterioridad - estar fuera de un espacio-.

- La posición en el espacio con relación a otro objeto —alrededor, al centro...-.

- La relación de más de un objeto dentro de un espacio. Hallamos la interioridad - un objeto dentro de otro-, la sección — un objeto parcialmente dentro de otro-, la contigüidad - dos objetos son limitantes - y la exterioridad — los objetos no tienen espacio en común-.

- La distancia entre dos objetos - cerca y lejos-.

- La medida del espacio, de forma numérica $-1,4,6 \ldots-\mathrm{y}$ de forma relacional a través de las matemáticas - pesado-ligero, grande-pequeño...-.

Igualmente, para Castro (2004), las experiencias expresadas mediante el reconocimiento y representación gráfica de acercamientos, separación, orden, entorno y continuidad representan experiencias de carácter topológico. En este tipo de representación, las transformaciones sufridas por una figura original son tan profundas y generales que alteran los ángulos, las longitudes, las rectas, las áreas, los volúmenes, los puntos y las proporciones. A pesar de ello, algunas relaciones o propiedades geométricas permanecen invariables. Según Castro (2004), las relaciones espaciales — que determinan la proximidad o acercamiento, la separación o alejamiento entre puntos y/o regiones, la condición de cierre de un contorno, la secuencia, la continuidad o la discontinuidad de líneas, superficies o volúmenes- son propiedades geométricas que se conservan en una transformación de carácter topológico.

En este orden de ideas, es necesario buscar estrategias que permitan reconocer los espacios, descubrirlos, expresarse e interpretarlos para orientarse en ellos. Por tal motivo, en esta investigación se busca utilizar los kenebo shipibo-conibo como una de ellas.

El kené es la forma de expresión de la cosmovisión shipibo-conibo, un arte del diseño ejercida y transmitida por las mujeres (Belaunde, 2012). Para el pueblo shipibo-conibo, el diseño -o kené-, que se refiere al pintado, bordado y tejido de diseños propios, es un arte que se transmite de generación en generación, principalmente de madres a hijas (Belaunde, 2012). Por esa razón, el conocimiento del significado simbólico de dichos diseños es de suma importancia, sobre todo para los niños, en especial para desarrollar competencias que definan su identidad y la noción de su ser en el contexto espacial y temporal. Como lo expresa González (2016), el kené no es considerado por el pueblo shipibo-conibo solo como una manifestación estética, sino que cumple una función como agente activo de protección y de manutención de su salud física y espiritual, de allí su importancia para este pueblo ancestral.

Los grupos indígenas que conforman la comunidad shipibo-conibo son un pueblo indígena perteneciente a la Amazonía peruana y de la familia lingüística pano. Este pueblo habita en los márgenes del río Ucayali y los afluentes Pisqui, Callería, Aguaytia y Pachitea, así como a orillas de los lagos Imiría y Yarina (Mansilla, 2019). Para el pueblo shipibo-conibo, los kenebos que se realizan sobre las telas, cerámica, el cuerpo y otros objetos son parte fundamental de su cultura. Son la representación de la relación del mundo material con el espiritual (Belaunde, 2012; Espino, 2018). El kené representa además el mantenimiento en el presente de las prácticas del pasado, expresado en el día a día. Además, es el vehículo para 
la comunicación de los vivos con los muertos, ya que en sus trazos se dibuja el camino que lleva hacia los ancestros (Morales \& Mujica, 2019). De lo anterior se deduce que, para los niños y niñas shipibo-conibo, conocer el significado de los diseños es parte importante no solo de su cultura, sino también del conocimiento que deben tener de su entorno o espacio, tanto natural, como social y espiritual, es decir de su cosmovisión (Brabec \& Mori, 2009).

Un aspecto clave de la polisemia del kené reside en la asociación de los grafismos con el concepto de cano - camino-. Según el pensamiento shipibo-conibo, los trazos plasman una armazón -canóa - de caminos por los que se movilizan los seres, que viajan y se comunican entre sí mientras transportan conocimientos, objetos y poderes (Belaunde, 2009).

La visión del kené para los shipibo-conibo es fundamental para entender su relación con lo natural, con lo espiritual y, por ende, con la noción de su ser en el espacio y el tiempo. Sin embargo, se pudo constatar que los niños y niñas que fueron escogidos para el estudio estaban en un nivel de aprendizaje en proceso, de acuerdo con su conocimiento sobre la $\mathrm{NE}$, lo que pudo estar influenciado por su corta edad, aun cuando es imprescindible para su cultura que la visión de su cosmovisión sea alimentada desde los primeros años de vida, dada su complejidad en concebir los espacios, seres del mundo y sus interrelaciones (Delgado, 2017).

Algunos elementos de la cultura shipibo-conibo han sido estudiados como parte fundamental del pueblo y también se han utilizado como estrategias pedagógicas. Entre ellas se puede mencionar el uso del canto para la enseñanza y la conservación de la lengua propia del pueblo (Mendivel et al., 2016). De igual forma, los juegos tradicionales de los shipibo-conibo se han utilizado como estrategias pedagógicas (Monteluisa et al., 2015). A pesar de que los diseños son considerados como los elementos más distintivos y que revisten de gran importancia para los shipibo-conibo, no existe evidencia de su uso como parte de las estrategias pedagógicas utilizadas en la educación intercultural que reciben los niños. Por ello, esta investigación se enfoca en utilizar el sentido físico y espiritual de los diseños como base para la comprensión de la NE, al usarlos como técnica de enseñanza en el taller dirigido por el docente.

En el desarrollo de la NE en niños y niñas, el taller como estrategia pedagógica es una técnica activa, con la cual se pueden conseguir resultados tangibles y en corto tiempo, ya que se trata de una forma didáctica enfocada no solo en enseñar, sino también en aprender, con base en una serie de actividades que los niños realizan de manera conjunta (Gutiérrez, 2009). De igual forma, se ha comprobado que los talleres pedagógicos como estrategia metodológica aplicada en el proceso de enseñanza-aprendizaje son instrumentos adecuados que ayudan a la investigación educativa, dado que en ellos se presenta la interacción entre los estudiantes y con el docente, lo cual integra la teoría y la práctica para facilitar la comprensión de las realidades mediante la interrelación del conocimiento y la acción (Aponte, 2015).

Según lo anterior, Suárez (2018) señala que se deben presentar alternativas para implementar una mejor educación, que respete la identidad, la cultura y la lengua de los pueblos amazónicos. En tal sentido, el objetivo es determinar la influencia de los kenebo shipibo-conibo sobre la NE topológica en niños de educación inicial. 


\section{Metodología}

La investigación se desarrolló con un enfoque cuantitativo y descriptivo. Se centró en aspectos observables y susceptibles de cuantificar con el uso de la metodología empírica analítica. Se utilizaron pruebas estadísticas para el análisis de datos. La muestra estuvo integrada por 48 niños de cinco años pertenecientes a la comunidad shipibo-conibo, quienes son estudiantes regulares de las instituciones educativas del nivel inicial $\mathrm{N}^{\circ} 470-\mathrm{B}$ de la Asociación Intercultural Yine y $\mathrm{N}^{\circ}$ 377-B Bena Jema del distrito de Yarinacocha, Provincia Coronel Portillo, Región Ucayali, Perú, durante el 2019. La muestra fue escogida de forma no probabilística y por conveniencias, ya que el nivel inicial en el ciclo II cuenta con niños de 3 a 5 años, de los que solo se escogieron los de 5 años. La muestra fue dividida en dos grupos de 24 niños: un grupo control (GC) y un grupo experimental (GE), que se formaron mediante asignación al azar de los sujetos. Para la medición de la NE, se aplicó una guía de observación que consistió en un listado de ítems por aspectos que orientan en la observación del desarrollo de la NE de los niños y niñas de cinco años. Este instrumento contenía 30 ítems, en escala: sí = 1 y no $=0$ puntos. En la tabla 1, se muestran las características de la muestra estudiada.

Tabla 1

Descripción de la muestra y distribución de los grupos de trabajo

\begin{tabular}{|c|c|c|c|c|c|}
\hline \multirow{2}{*}{ Grupo } & \multirow{2}{*}{ Institución Educativa } & \multirow{2}{*}{ Edades } & \multicolumn{2}{|c|}{ Sexo } & \multirow{2}{*}{$N^{\circ}$ de niños y niñas } \\
\hline & & & M & $\mathbf{F}$ & \\
\hline GE & $\begin{array}{l}\mathrm{N}^{\circ} 470 \text {-B Asoc. Intercultural } \\
\text { Yine }\end{array}$ & 5 años & 11 & 13 & 24 \\
\hline GC & N³77-B Bena Jema & 5 años & 13 & 11 & 24 \\
\hline \multicolumn{3}{|c|}{ Total } & 24 & 24 & 48 \\
\hline
\end{tabular}

El GE fue sometido a una intervención en la institución por treinta minutos durante una semana, mediante talleres de kenebo shipibo-conibo, ajustados a la planificación del docente, los cuales se enfocaron en diferentes actividades tales como pintura, dibujos, mapas, canto y juegos. Los juegos de reconocimiento del espacio buscan ayudar al niño a descubrirlo y enunciarse en él. En este sentido, en la tabla 2 se describe uno de los juegos utilizados, llamado la Danza de la Boa. El resto de las actividades fue realizado con diferentes kenebos como guía. Prevalecieron los juegos dirigidos por los docentes en los que estos diseños se asemejan a los juegos de la población shipibo-conibo, con los cuales los niños reconocen delante, detrás, izquierda y derecha.

Las actividades se realizaron con el propósito de potenciar aspectos de la cultura shipibo-conibo como expresión artística, sistema de valores culturales, representación de los diseños y simbolización de la relación con la naturaleza y los valores productivos. Se toman expresiones que permitan la conjunción de los sentidos visuales, auditivos, táctiles y olfativos. La incorporación de diseños en la enseñanza, tal como señala Suárez (2018), demanda en principio planificar un modelo pedagógico que permita aprovecharlos como portadores de sapiencias. Enseñar a mirar implica interpretar, vislumbrar y aplicar procesos cognitivos para entender el mensaje visual. La efectividad de la intervención se midió a través de su influencia sobre la NE, que se define como la construcción del concepto de espacio del ser humano, como producto de lo vivido y experimentado por sí mismo y por su cuerpo (García et al., 2015). 
Tabla 2

Descripción de la Danza de la Boa

\begin{tabular}{|c|c|}
\hline & Danza de la Boa \\
\hline \multicolumn{2}{|c|}{ Homenaje a "Ronin", la serpiente cósmica, el río que marca la vida del pueblo shipibo-konibo } \\
\hline $\begin{array}{l}\text { Objetivo: Reforzar las } \\
\text { NE }\end{array}$ & Procedimiento \\
\hline $\begin{array}{l}\text { Materiales } \\
\text { Sillas } \\
\text { Música }\end{array}$ & $\begin{array}{l}\text { Es un juego de orientación espacial adaptado del baile de sillas. } \\
\text { Se hace una serpiente - tomando como ejemplo un kenet representativo de la } \\
\text { misma- en forma de semicírculo hecha por sillas colocadas hacia fuera. Siem- } \\
\text { pre hay una menos que el número de niños. Los niños cantan y bailan alrede- } \\
\text { dor de las sillas. En un momento la maestra dice "jalto!" y los niños deberán } \\
\text { sentarse. El niño que queda de pie sale del juego y se retira una silla. El juego } \\
\text { continúa hasta que el último jugador se siente en la silla que queda, quien será } \\
\text { el ganador. La variante en este juego es que la maestra dirigirá los movimientos } \\
\text { para que con el juego consoliden las NE. Por ejemplo, ella dirá "ahora bailamos } \\
\text { yendo hacia atrás, con las manos arriba, hacia abajo, derecha e izquierda". }\end{array}$ \\
\hline
\end{tabular}

Para la medición de la NE, se aplicó una guía de observación. Se desarrollaron seis dimensiones de la NE, cada una con cinco ítems respectivamente:

- esquema corporal: toma de conciencia de su imagen corporal o representación de su propio cuerpo.

- coordinación: la interacción, el funcionamiento del sistema nervioso central y la musculatura esquelética durante el ejercicio.

- ubicación temporal: orientación del propio cuerpo en cuanto al mundo; es saber dónde estoy y dónde está lo que me rodea.

- ubicación espacial: conocer y determinar la posición del propio cuerpo con relación al espacio.

- percepción: modo en que el cerebro interpreta la información que llega a través de los sentidos - vista, oído, olfato, gusto y tacto-.

- lateralidad: preferencia que cada niño muestra por un lado de su cuerpo; este fenómeno se debe al predominio de uno de los hemisferios cerebrales.

Tabla 3

Baremo de puntuación de la guía de observación y la prueba gráfica

\begin{tabular}{|c|c|c|c|c|}
\hline Variable & Dimensiones & $\mathrm{N}^{\circ}$ de ítems & Peso & Estimación \\
\hline \multirow{6}{*}{$\mathrm{NE}$} & D1: esquema corporal & 5 & \multirow{6}{*}{5} & \multirow{6}{*}{$\begin{array}{l}4-5 \\
2-3 \\
0-1\end{array}$} \\
\hline & D2: coordinación & 5 & & \\
\hline & D3: ubicación temporal & 5 & & \\
\hline & D4: ubicación espacial & 5 & & \\
\hline & D5: percepción & 5 & & \\
\hline & D6: lateralidad & 5 & & \\
\hline \multirow{3}{*}{ Baremo } & & & & $21-30$ \\
\hline & 6 & 30 & 30 & $11-20$ \\
\hline & & & & $0-10$ \\
\hline
\end{tabular}

Para facilitar la observación, se aplicó también una prueba gráfica basada en kenebos shipibo-conibo para evaluar nociones de orientación espacial, de la cual se extrajo 
la información que se registra en la guía de observación. La estructuración del baremo de puntuación referente a los aspectos considerados en la prueba gráfica con elementos de kenebo se muestra en la tabla 3. Cabe señalar que los docentes también son parte de la comunidad shipibo-conibo y, por ende, participaron en la implementación de los instrumentos. Luego de una asesoría del investigador, la guía de observación fue aplicada por los docentes responsables de manera simultánea con los grupos, en el mismo espacio. El investigador permaneció como observador.

El puntaje apreciativo de acuerdo con el baremo se estableció como sigue.

Para las dimensiones:

- Puntaje de $0-1=$ inicio de aprendizaje.

- Puntaje de 2-3 = en proceso de aprendizaje.

- Puntaje de 4-5 = aprendizaje logrado.

Para la NE:

- Puntaje de 1 a $10=$ NE en inicio del aprendizaje.

- Puntaje de $11-20=$ NE en proceso de aprendizaje.

- Puntaje de 21 - 30 = NE logrado.

El diseño de la investigación fue cuasiexperimental con pre- y postest e intervención al GE, de acuerdo con el esquema mostrado en la figura 1.

\section{Figura 1}

Diseño experimental aplicado

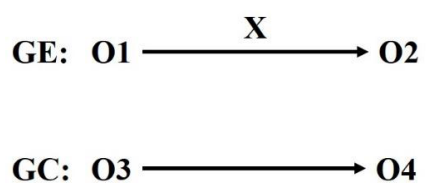

Nota. GE: grupo experimental; GC: grupo control; O1: pretest aplicado al grupo experimental; O3: pretest aplicado al grupo control; O2: postest aplicado al grupo experimental; O4: postest aplicado al grupo control; X: aplicación de la intervención con talleres de diseño.

El tratamiento estadístico de los datos se realizó mediante métodos descriptivos, basados en medidas de tendencia central — media, mediana y moda - y medidas de variabilidad — desviación estándar, varianza y coeficiente de variación-; y en métodos de comparación no paramétricos de U de Mann-Whitney y de comparación de distribuciones de Kolmogorov-Smirnov. Lo anterior se realizó para establecer la influencia de la intervención con los talleres de diseño shipiboconibo sobre las competencias de los estudiantes del GE respecto a la NE. Para todos los análisis estadísticos, se utilizó el paquete Statgraphics Centurion XVII.

\section{Resultados}

Los resultados del análisis estadístico-descriptivo del pretest aplicado a los dos grupos se muestran en la tabla 4 , donde se observan los resultados por dimensiones y el total de la variable NE. 
Tabla 4

Resultados de la estadística descriptiva del pretest aplicado a los grupos control y experimental

\begin{tabular}{lccccccccccccccc} 
& \multicolumn{3}{c}{} & \multicolumn{1}{c}{ D1 } & \multicolumn{2}{c}{ D2 } & \multicolumn{2}{c}{ D3 } & \multicolumn{2}{c}{ D4 } & \multicolumn{2}{c}{ D5 } & \multicolumn{2}{c}{ D6 } & \multicolumn{2}{c}{ NE } \\
\cline { 2 - 14 } & GC & GE & GC & GE & GC & GE & GC & GE & GC & GE & GC & GE & GC & GE \\
\hline Media & 1,3 & 3,2 & 2,5 & 3,2 & 2,0 & 3,1 & 2,6 & 3,2 & 2,6 & 3,3 & 1,3 & 3,3 & 12,3 & 19,2 \\
Mediana & 1 & 3 & 2,5 & 3 & 2 & 3 & 3 & 3 & 2 & 3 & 1 & 3 & 12 & 18 \\
Moda & 3 & 4 & 4 & 4 & 4 & 4 & 5 & 4 & 5 & 4 & 3 & 4 & 17 & 23 \\
VAR & 1,7 & 0,6 & 0,7 & 0,5 & 1,7 & 0,6 & 1,3 & 0,7 & 1,9 & 0,5 & 0,9 & 0,5 & 14,7 & 8,2 \\
DE & 1,3 & 0,8 & 0,8 & 0,7 & 1,3 & 0,8 & 1,1 & 0,8 & 1,4 & 0,7 & 1,0 & 0,7 & 3,8 & 2,9 \\
CV \% & 98,1 & 24,0 & 32,8 & 22,2 & 66,5 & 25,2 & 44,1 & 25,9 & 53,4 & 20,8 & 73,9 & 20,9 & 31,2 & 14,9 \\
\hline
\end{tabular}

En general, las dimensiones medidas en el GC mostraron valores promedio entre 1 y 3 , lo que indica, según el baremo establecido, que los niños están inicialmente en el rango entre inicio y en proceso de aprendizaje de las NE, lo que se corrobora con el valor medio de la variable, el cual estuvo en el rango correspondiente al nivel en proceso de aprendizaje. En el caso del GE, las dimensiones presentaron valores medios aproximados de 3, lo que significa que inicialmente este grupo está en el nivel correspondiente a en proceso de aprendizaje de las NE, lo cual se observa también en la variable total, cuya media se ubicó entre 11 y 20. En ambos grupos el puntaje que más se repitió — moda - en las dimensiones se ubicó mayormente en 4, lo que indica que, si bien - en general- los grupos están en proceso de aprendizaje, existe un porcentaje importante de niños con NE logrado inicialmente. La desviación estándar, la varianza y el coeficiente de variación indican que existe mayor variabilidad de los puntajes en el GC, en comparación con el GE.

La distribución de los niveles de ambos grupos se puede observar en la figura 2, en la que se muestra que el mayor porcentaje de niños para ambos grupos está en el nivel de aprendizaje en proceso, respecto a su conocimiento sobre las NE.

\section{Figura 2}

Distribución de los niveles iniciales - pretest-de NE de los niños

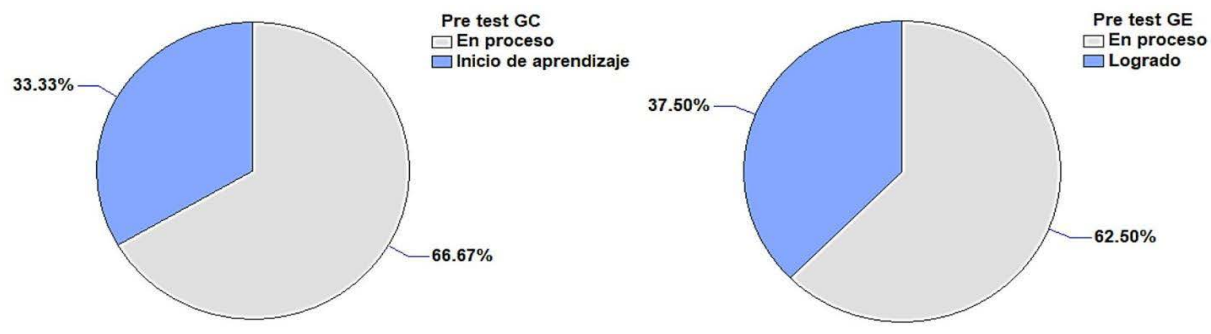

Se observa también que en el GE no hay niños ubicados en el nivel de inicio de aprendizaje y los porcentajes son similares. El GE muestra un porcentaje mayor de niños con la competencia lograda al inicio de la investigación.

Luego de la intervención del GE con los talleres de diseño shipibo-conibo, se aplicó el instrumento - postest- y los resultados se muestran en la tabla 5 .

Se observa que el GC presenta un rango de puntajes medios entre 1,8 y 2,6 en las dimensiones, lo cual indica que se mantiene entre los niveles inicial y en proceso de aprendizaje. La media total de 13,4 ubica a este grupo en el nivel en proceso de aprendizaje, 
respecto a la variable NE después de la aplicación del postest. Con relación al GE, se observa que todas las dimensiones se ubican en el nivel logrado -45- con un valor menor para la ubicación espacial, con 4,3. El valor medio total de la variable NE para el GE, se ubicó en el nivel logrado, lo que sugiere una mejora importante respecto al valor inicial arrojado por el pretest. Los parámetros estadísticos de variabilidad indican que en el GE se obtuvieron resultados más homogéneos, sobre todo con relación al coeficiente de variación, el cual fue de $6,5 \%$, bastante bajo en comparación con el 32,8 \% del GC.

Tabla 5

Resultados de la estadística descriptiva del postest aplicado a los grupos control y experimental

\begin{tabular}{|c|c|c|c|c|c|c|c|c|c|c|c|c|c|c|}
\hline & \multicolumn{2}{|c|}{ D1 } & \multicolumn{2}{|c|}{ D2 } & \multicolumn{2}{|c|}{ D3 } & \multicolumn{2}{|c|}{ D4 } & \multicolumn{2}{|c|}{ D5 } & \multicolumn{2}{|c|}{ D6 } & \multicolumn{2}{|c|}{ NE } \\
\hline & GC & GE & GC & GE & GC & GE & GC & GE & GC & GE & GC & GE & GC & GE \\
\hline Media & 1,9 & 4,6 & 2,4 & 4,8 & 2,1 & 4,9 & 2,6 & 4,3 & 2,6 & 4,9 & 1,8 & 4,7 & 13,4 & 28,3 \\
\hline Mediana & 2 & 5 & 2 & 5 & 2 & 5 & 3 & 5 & 2 & 5 & 2 & 5 & 14 & 28,5 \\
\hline Moda & 3 & 5 & 4 & 5 & 4 & 5 & 4 & 5 & 5 & 5 & 3 & 5 & 18 & 30 \\
\hline VAR & 1,8 & 1,0 & 0,6 & 0,2 & 1,9 & 0,1 & 1,1 & 0,9 & 1,9 & 0,1 & 0,9 & 0,4 & 19,3 & 3,4 \\
\hline $\mathrm{DE}$ & 1,3 & 1,0 & 0,8 & 0,5 & 1,4 & 0,2 & 1,1 & 0,9 & 1,4 & 0,2 & 1,0 & 0,6 & 4,4 & 1,9 \\
\hline CV \% & 68,1 & 22,2 & 32,4 & 10,0 & 67,8 & 4,1 & 41,0 & 22,2 & 53,4 & 4,1 & 54,5 & 13,3 & 32,8 & 6,5 \\
\hline
\end{tabular}

La distribución de los niveles en el postest mostró que, en el caso del GE, todos los niños se ubicaron en el nivel logrado - 100\%-. Sin embargo, en el caso del GC, como se observa en la figura 3, el $75 \%$ se ubicó en el nivel en proceso y $25 \%$ en nivel inicio del aprendizaje.

\section{Figura 3}

Distribución de niveles de NE de los niños del GC luego del postest

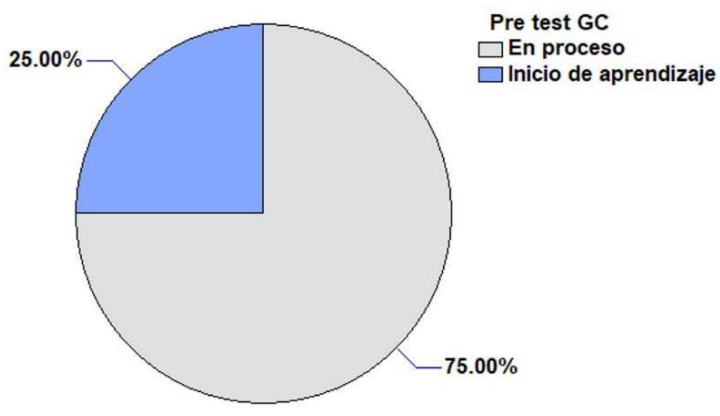

Antes de la comparación de los grupos con respecto a las notas obtenidas en el pretest y el postest, se analizó el supuesto de normalidad de los datos mediante el test de Shapiro-Wilk, debido a que este test es recomendado para muestras pequeñas, de menos de cincuenta datos (Cabrera et al., 2017), con la finalidad de establecer si la comparación sería con estadística paramétrica o no paramétrica. Los resultados se muestran en la tabla 6.

\section{Tabla 6}

Resultados de la prueba de bondad de ajuste de Shapiro-Wilk

\begin{tabular}{ccc}
\hline Datos & Estadístico W de Shapiro-Wilk & Valor-p \\
\hline Pretest GC & 0,910411 & 0,0354521 \\
Postest GC & 0,918322 & 0,0537866 \\
Pretest GE & 0,912502 & 0,0395753
\end{tabular}


Como se observa, con excepción de los resultados del postest del GC, los demás resultados presentan valores $\mathrm{p}<0,05$, lo que indica que se puede descartar la hipótesis de que los datos provienen de una distribución normal. Aun cuando los resultados del postest GC tienen valor $\mathrm{p}>0,05$, están bastante cerca de este valor límite. Además, no se pueden aplicar pruebas paramétricas, ya que solo serían aplicables a esta muestra e invalidarían los demás. De acuerdo con lo anterior, se aplicaron pruebas de comparación no paramétricas, las cuales son aplicables a todos los resultados obtenidos. En la tabla 7 se muestra la comparación de medianas con la prueba U de Mann-Whitney, con nivel de confianza de $95 \%$.

Tabla 7

Resultados de la prueba de comparación de medias U de Mann-Whitney

\begin{tabular}{ccccc}
\hline Grupos comparados & Medianas & Rango promedio & U de Mann-Whitney & Valor-p \\
\hline Pretest GC & 12,0 & 22,23 & 342,5 & 0,262063 \\
Postest GC & 14,0 & 26,77 & & \\
\hline Pretest GE & 18,0 & 12,75 & 570,0 & 0,000000 \\
Postest GE & 28,5 & 36,25 & & \\
\hline
\end{tabular}

En la tabla 7 se observa que, al comparar los puntajes del GC en el pre- y el postest, se obtiene un valor $\mathrm{p}>0,05$, lo que indica que no hay diferencia estadísticamente significativa entre las medianas con un 95,0 \% de confianza. Por otro lado, en el caso del GE, al comparar los puntajes entre el pre- y el postest, el valor $\mathrm{p}<0,05$ indica que sí existe diferencia estadísticamente significativa, con un nivel de confianza de 95,0\%, lo que demuestra que la intervención del grupo con los talleres de diseño shipibo-conibo influyó de forma positiva en el puntaje obtenido por los niños.

Para analizar las diferencias entre las distribuciones de las calificaciones, se utilizó la prueba de Kolmogorov-Smirnov, la cual, en el caso del GC, arrojó un valor del estadístico $\mathrm{K}-\mathrm{S}$ bilateral $=0,866025$, con un valor $\mathrm{p}=0,448738$, que, al ser mayor que 0,05 , indica que no existe diferencia significativa entre las dos distribuciones. Por lo tanto, se puede decir que las calificaciones del GC no difieren en su tendencia entre el pre- y el postest, con un nivel de confianza de 95,0 \%. El comportamiento gráfico se puede observar en la figura 4 .

\section{Figura 4}

Gráficas de comparación de distribuciones para el GC. (a) Comparación de distribución de frecuencias. (b) Comparación de densidad suavizada

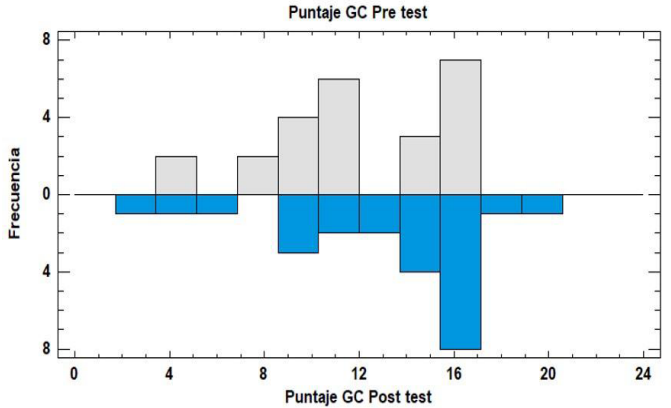

(a)

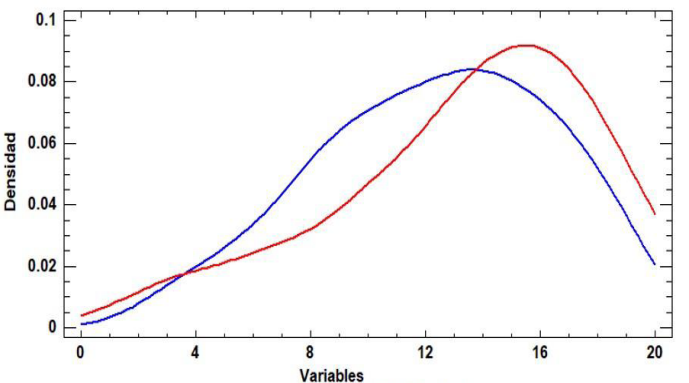

(b) - Puntaje GC Pre test 
En las gráficas de la figura 4 se observa claramente cómo las distribuciones de las calificaciones del GC se comportan de forma equivalente tanto en el pretest como en el postest. Su distribución de frecuencias es equivalente (a). Solo con una pequeña diferencia en el postest, se obtuvieron dos calificaciones entre 17 y 20. Con relación a las curvas de densidad suavizada, las tendencias fueron similares, con una ligera diferencia y una mayor densidad en las mayores calificaciones para el postest.

En el GE, se obtuvo que un estadístico K-S bilateral $=3,4641$ y valor $\mathrm{p}=0,000000$, que demuestran que las distribuciones de las calificaciones en el pretest y el postest presentan diferencias estadísticamente significativas, con un nivel de confianza de 95,0 \%. Por ello, las calificaciones en el postest se comportan y se distribuyen de forma diferente a las que se obtuvieron en el pretest. El comportamiento gráfico se observa en la figura 5.

\section{Figura 5}

Gráficas de comparación de distribuciones para el GE. (a) Comparación de distribución de frecuencias. (b) Comparación de densidad suavizada

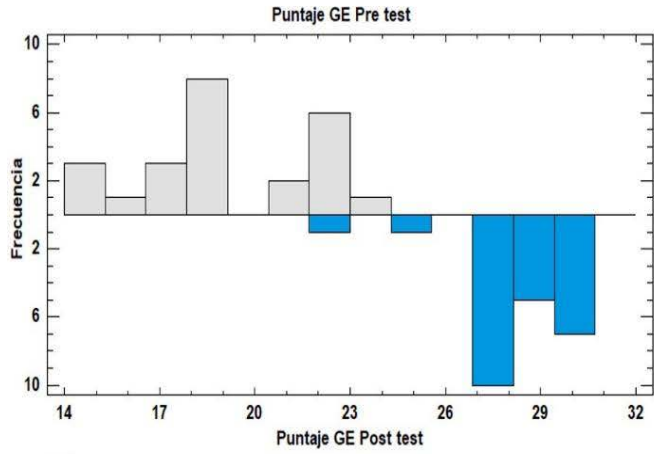

(a)

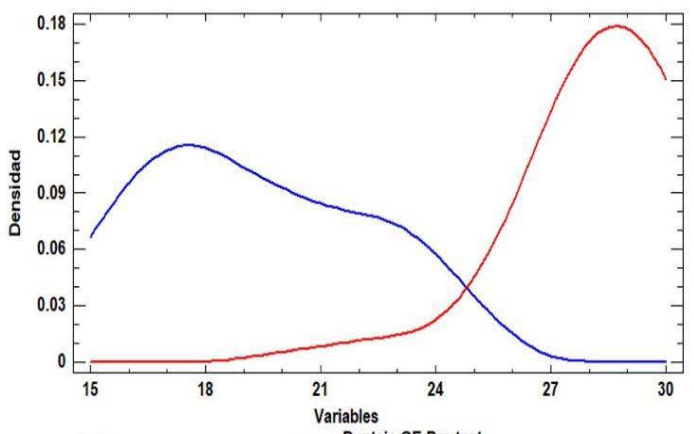

(b)

En la figura 5 (a) se observa que las distribuciones de las frecuencias en el pretest se ubican en lo más bajo de la escala, es decir entre las calificaciones menores a 25. Por el contrario, en el postest la distribución de frecuencias se acumula hacia los puntajes mayores, es decir, entre 27 y 30, lo que demuestra la diferencia entre ambos test. En la gráfica de comparación de las densidades suavizadas (b), se nota claramente cómo en el pretest las mayores densidades - de acuerdo con las calificaciones- se acumulan en los puntajes menores de la escala y van disminuyendo a medida que se avanza en la misma. Por el contrario, en el postest las calificaciones se acumulan más densamente en lo alto de la escala de medida y disminuyen hacia los niveles más bajos. Lo anterior indica que las diferencias en las distribuciones se deben a un aumento significativo en las calificaciones del GE luego de la intervención con los talleres de diseño shipibo-conibo.

\section{Discusión}

La visión del kené para los shipibo-conibo es fundamental para entender su relación con lo natural y lo espiritual y, por ende, con la noción de su ser en el espacio y tiempo. Sin embargo, se pudo constatar que los niños y niñas que fueron escogidos para el estudio estaban en un nivel de aprendizaje en proceso, de acuerdo con su conocimiento sobre la $\mathrm{NE}$, lo que pudo estar influenciado por su corta edad, aun cuando es imprescindible para su cultura que su cosmovisión sea alimentada desde los primeros años de vida, dada su 
complejidad en concebir los espacios, los seres del mundo y sus interrelaciones (Delgado, 2017). El resultado inicial obtenido, respecto a las competencias de los niños de NE, coincide con el reportado por García et al. (2015), quienes concluyeron que la NE en niños de educación inicial se va fortaleciendo con el tiempo y que la misma depende de cómo se vaya desarrollando la organización del entorno en la que los niños se desarrollan.

La NE en niños es un concepto que, a juicio de Sánchez y Benítez (2014), es complejo de enseñar y de aprender, por lo que se debe abordar a partir de los primeros años de edad, es decir desde la fase de educación infantil, puesto que esta es una etapa de obtención "crítica" para el desarrollo evolutivo normal. Un valor adicional reposa en la posibilidad de consolidar el entendimiento de esas nociones, además del cumplimiento práctico de sus ideas respecto a tiempo y a espacio —fuera, adentro, arriba, abajo, entre otras-.

$\mathrm{Al}$ crecer, los niños van adquiriendo más conocimientos sobre la NE. En el caso de los pertenecientes a etnias originarias, como los shipibo-conibo, deben visualizar el concepto basado en la cosmovisión propia de su pueblo. Por lo tanto, la intervención temprana con estrategias que les permitan adquirir mejor el conocimiento es fundamental para su desarrollo educativo y personal. En este sentido, se ha determinado la efectividad del uso de estrategias basadas en el componente gráfico para mejorar la NE y la noción de tiempo de niños en edad inicial. En investigaciones como la de Weist (2002), se concluye que la asociación del espacio con dibujos genera una mayor comprensión por parte de niños con edades entre 3 y 8 años.

En la cultura shipibo-conibo, los diseños o trazos utilizados en textiles y cerámica no solo representan el espacio terrenal, sino también el espiritual. Los diseños son una expresión de la creatividad shipibo-conibo y simbolizan la interacción del hombre con el mundo material y las fuerzas invisibles de la naturaleza (Odland \& Feldman, 2010), por lo que el uso de dichos trazos en el conocimiento y desarrollo de la NE de los niños es una estrategia que permite mejorar las competencias de los niños. Esto se corrobora con los resultados obtenidos, donde, al aplicar al GE los talleres de kenebo, se logró una mejora significativa en el nivel de conocimiento, lo que llevó a que la totalidad de los niños mostraran el logro de las competencias. La efectividad del uso de diseños para fortalecer la NE de niños también fue observada por Villegas et al. (2013), quienes concluyeron que se pueden abordar con actividades que les permitan a los niños reconocer superficies, figuras y características de sus elementos. Con ello, se desarrollan NE proyectivas con un nivel del realismo intelectual $\mathrm{y}$ visual.

La representación de figuras y formas propias del diseño artístico y cultural permitió el desarrollo y el logro de las competencias vinculadas a la NE de los niños y niñas de la muestra. López et al. (2016) coinciden en afirmar que el conocimiento de las figuras, trazos y manifestaciones artísticas desarrollan capacidades de percepción de formas y espacio, que a su vez mejoran el análisis y la comprensión de las relaciones entre estos conceptos. Lo anterior se puede sustentar en el análisis estadístico realizado, el cual demostró que el aumento en las calificaciones luego de la intervención con los talleres de diseño en el GE fue significativo. Así, se comprueba la efectividad de la estrategia aplicada. Los talleres como estrategia didáctica, independientemente del contenido impartido, han demostrado ser eficientes, dado que son una alternativa de enseñanza-aprendizaje cuya función es traer 
elementos de la realidad al aula de clases (Aponte, 2015).

Otros elementos culturales del pueblo shipibo-conibo también han demostrado ser eficientes elementos pedagógicos, como el caso de los cantos, estudiados por Mendivel et al. (2016), quienes concluyeron que utilizados como estrategia didáctica favorecen de forma significativa la enseñanza y la recuperación de la lengua de este pueblo originario. Por su parte, Monteluisa et al. (2015) también concluyeron que el uso de juegos tradicionales y propios de la cultura shipibo-conibo es una estrategia pedagógica que influye positivamente en el aprendizaje de los niños. Lo anterior indica que, al combinar diferentes elementos culturales y cotidianos en la educación intercultural, se pueden obtener resultados positivos y significativos, algo que fue discutido igualmente por Cépeda et al. (2019), quienes indican que los docentes de las escuelas interculturales deben reconocer las relaciones entre grupos étnicos y sus culturas, para establecer verdaderas relaciones interculturales. Esto implica que en la enseñanza deben estar presentes elementos culturales y étnicos que contribuyan a la profundización y a la consolidación de las concepciones y prácticas que lleven a una interculturalidad con perspectiva crítica.

\section{Conclusiones}

Belaunde (2009) señala que el principal equívoco cuando se aborda el estudio del kené es querer interpretar su significado como si los trazos geométricos, las curvas y las líneas que forman los diseños fuesen estrictamente representaciones figurativas de alguna cosa. Hay un aspecto figurativo en el kené en la medida que los trazos son llamados con nombres que representan las actividades en las que se ponen en práctica.

Los resultados mostraron que los niños y niñas de cinco años de la comunidad shipiboconibo que presentaban un nivel inicial y en proceso de capacidad cognitiva de NE, una vez intervenidos a través de las actividades desarrolladas en el taller basado en los kenebo, mejoraron en este aspecto de forma efectiva, ya que exhibieron una mejor noción espacial y se ubicaron en un nivel de logro de las capacidades requeridas para la interpretación del espacio topológico. Piaget (1985) expone que las NE se acrecientan con el tiempo según las actividades ejecutadas por los niños. Es decir, esta noción es un constructo y no algo que viene dado.

Asimismo, se corrobora lo dicho por Belaunde (2009), cuando demuestra que la enseñanza de los principios artísticos, culturales y espirituales de los kenebo - producto de un conocimiento ancestral- desempeña un papel importante en la percepción y en la interpretación por parte de los infantes sobre el espacio que los rodea y su relación con sus raíces y con la cosmovisión del pueblo shipibo-conibo.

El conocimiento y la interpretación del sentido cultural y espiritual de los kenebo shipibo-conibo, combinado con las actividades pedagógicas de talleres, son una estrategia válida para generar conocimiento significativo y competencias específicas. Por ello, se debe seguir indagando en este campo, con la finalidad de mejorar y de profundizar los contenidos curriculares de la educación intercultural bilingüe, para contribuir a la preservación del acervo cultural y espiritual de los pueblos originarios en Perú. 


\section{Referencias}

Aponte, R. (2015). El taller como estrategia metodológica para estimular la investigación en el proceso de enseñanza-aprendizaje en la educación superior. Boletín Virtual, 4(10), 49-55. https://dialnet.unirioja.es/servlet/articulo?codigo $=6232367$

Belaunde, L. (2009). Kené: arte, ciencia y tradición en diseño. Instituto Nacional de Cultura.

Belaunde, L. (2012). Diseños materiales e inmateriales: la patrimonialización del kené shipibo-konibo y de la ayahuasca en el Perú. Mundo Amazónico, 3, 123-146. https:// revistas.unal.edu.co/index.php/imanimundo/article/view/28715

Brabec, B., \& Mori, L. (2009). La corona de la inspiración. Los diseños geométricos de los Shipibo-Konibo y sus relaciones con cosmovisión y música. Indiana, (26), 105-134. https://journals.iai.spk-berlin.de/index.php/indiana/article/view/1969

Cabrera, G., Zanazzi, J., Zanazzi, J., \& Boaglio, L. (2017). Comparación de potencias en pruebas estadísticas de normalidad, con datos escasos. Revista de la Facultad de Ciencias Exactas, Físicas y Naturales, 4(2), 47-52. https://revistas.unc.edu.ar/index.php/FCEFyN/ article/view/16775

Castro, J. (2004). El desarrollo de la noción de espacio en el niño de Educación Inicial. Acción Pedagógica, 13(2), 162-170. https://n9.cl/uyrf1

Cépeda, N., Castro, D., \& Lamas, P. (2019). Concepciones de interculturalidad y práctica en aula: estudio con maestros de comunidades shipibas en el Perú. Educación, 28(54), 61-86. http://dx.doi.org/10.18800/educacion.201901.004

Delgado, G. (2017). El malestar en la cultura shipibo-konibo [Tesis doctoral, Universidad Complutense de Madrid]. E-Prints Complutense. https://eprints.ucm.es/id/ eprint/43274/1/T38923.pdf

Espino, G. (2018). Literatura indígena amazónica shipibo-conibo y el kené de la palabra de Lastenia Canayo. Estudios Filológicos, 62, 247-267. http://dx.doi.org/10.4067/S007117132018000200247

Fernández, J., \& Ramiro, E. (2015). El concepto espacio en educación infantil [Trabajo final de grado, Universitat Jaume I]. Repositori Universitat Jaume I. http://repositori.uji.es/ xmlui/handle/10234/159051

García, M., Villegas, M., \& González, F. (2015). La noción del espacio en la primera infancia: un análisis desde los dibujos infantiles. Revista Paradigma, 36(2), 225-245. http:// ve.scielo.org/pdf/pdg/v36n2/art11.pdf

González, P. (2016). La tradición de arte chamánico shipibo-conibo (Amazonía peruana) y su relación con la cultura diaguita chilena. Boletín del Museo Chileno de Arte Precolombino, 21(1), 27-47. https://dx.doi.org/10.4067/S0718-68942016000100003

Gutiérrez, D. (2009). El taller como estrategia didáctica. Razón y Palabra, 66, 1-4. http:// www.redalyc.org/articulo.oa?id=199520908023

López, I., San Pedro, B., San Pedro, J., \& González, C. (2016). Enseñar dibujo y representación espacial en los estudios de grado en Educación Infantil y Primaria desde 
la Educación Artística. Revista de Psicología y Educación, 11(1), 61-80. http://www. revistadepsicologiayeducacion.es/pdf/136.pdf

Mansilla, J. (2019). Olivia Arévalo Lomas: una ventana desde el infinito asesinato del canto sagrado Shipibo-Konibo-Xeteba. Revista Historia de la Educación Latinoamericana, 21(33), 17-34. https://doi.org/10.19053/01227238.9909

Mendivel, R., Arias, J., \& Aguilar, R. (2016). Estrategia didáctica basada en cantos shipibos para recuperar la lengua shipibo-konibo en niños de Educación Inicial, Ucayali. Apuntes de Ciencia y Sociedad, 6(2), 143-149. http://dx.doi.org/10.18259/acs.2016023

Monteluisa, G., Valera, V., Frisancho, F., Frech, H., \& Delgado, E. (2015). Non Tsinitibo: juegos del pueblo shipibo-konibo y su uso pedagógico. Educación, 24(47), 49-68. http:// revistas.pucp.edu.pe/index.php/educacion/article/view/14241

Morales, D., \& Mujica, A. (2019). La arqueología y el mito de origen de los shipibo-conibo de la Amazonía peruana. Investigaciones Sociales, 22(40), 85-96. http://dx.doi.org/10.15381/ is.v22i40.15888

Morales, E. (2014). Espacio educativo de aprendizaje para el nivel inicial [Informe previo a Licenciatura, Universidad Rafael Landívar]. Crai Landívar Red de Bibliotecas. http:// biblio3.url.edu.gt/Tesario/2014/05/84/Morales-Eugenia2.pdf

Odland, J., \& Feldman, N. (2010, 1 de enero). Shipibo textilepractices 1950-2010 [Conferencia]. Textile Society of America Symposium Proceedings. University of Nebraska-Lincoln, Nebraska, Estados Unidos.

Piaget, J. (1985). Epistemología y psicología. Planeta DeAgostini.

Rimassa, C., \& Fernández-Silva, S. (2014). Conceptualización del espacio y su relación con el desarrollo cognitivo: un estudio piloto en el español de Chile. Alpha (Osorno), 38, 137154. http://dx.doi.org/10.4067/S0718-22012014000100010

Sánchez, J., \& Benítez, J. (2014). Nociones espacio-temporales y bimodal: análisis de una implementación educativa para alumnado de 3 años. International Journal of Developmental and Educational Psychology, 3(1), 165-177. https://www.redalyc.org/ pdf/3498/349851785017.pdf

Suárez, R. (2018, 09-12 de diciembre). El proceso de enseñanza /aprendizaje mediados por las iconografías del pueblo shipibo-conibo [Ponencia]. 31 ${ }^{\text {a }}$ Reunião Brasileira de Antropologia, Universidade de Brasília, Brasília/DF, Brasil.

Vargas, J. (2018). 87 años de educación inicial en el Perú. https://departamento.pucp.edu.pe/ educacion/noticias/87-anos-educacion-inicial-peru/

Villegas, M., Medina, M., García, M., \& González, F. (2013, 16-18 de octubre). Las nociones espaciales en educación infantil. Un estudio diagnóstico [Conferencia]. VI Congresso Internacional de Ensino da Matemática, Canoas, Brasil.

Weist, R. (2002). Temporal and spatial concepts in child language: conventional and configurational. Journal of Psycholinguistic Research, 31, 195-210. https://doi. org/10.1023/A:1015522711549 